\title{
Hyperentanglement in structured quantum light
}

\author{
Francesco Graffitti $\odot,{ }^{1, *}$ Vincenzo D’Ambrosio, ${ }^{2, \dagger}$ Massimiliano Proietti, ${ }^{1}$ Joseph Ho $\odot,{ }^{1}$ Bruno Piccirillo, ${ }^{2}$ \\ Corrado de Lisio $\odot,{ }^{2,3}$ Lorenzo Marrucci $\odot,{ }^{2}$ and Alessandro Fedrizzi ${ }^{1}$ \\ ${ }^{1}$ Institute of Photonics and Quantum Sciences, School of Engineering and Physical Sciences, Heriot-Watt University, \\ Edinburgh EH14 4AS, Scotland, United Kingdom \\ ${ }^{2}$ Dipartimento di Fisica, Università di Napoli Federico II, Complesso Universitario di Monte Sant'Angelo, Via Cintia, 80126 Napoli, Italy \\ ${ }^{3}$ Consiglio Nazionale delle Ricerche-SPIN U.O.S. di Napoli, Via Cintia 2, 80126 Napoli, Italy
}

(Received 15 May 2020; accepted 16 November 2020; published 9 December 2020)

\begin{abstract}
Entanglement in high-dimensional quantum systems, where one or more degrees of freedom of light are involved, offers increased information capacities and enables new quantum protocols. Here, we demonstrate a functional source of high-dimensional, noise-resilient hyperentangled states encoded in time-frequency and vector vortex structured modes, which in turn carry single-particle entanglement between polarization and orbital angular momentum. Pairing nonlinearity-engineered parametric downconversion in an interferometric scheme with spin-to-orbital-angular-momentum conversion, we generate highly entangled photon pairs at telecom wavelength that we characterize via two-photon interference and quantum state tomography, achieving near-unity visibilities and fidelities. While hyperentanglement has been demonstrated before in photonic qubits, here we present a rich entanglement structure involving spectrally and spatially structured light, where three different forms of entanglement coexist in the same biphoton state.
\end{abstract}

DOI: 10.1103/PhysRevResearch.2.043350

\section{INTRODUCTION}

Photonic platforms are a natural choice for many quantum applications owing to their advantages as low-noise quantum systems with high-fidelity control and suitability for long-distance transmission. The ubiquity of binary encoding in many proof-of-principle demonstrations, even for contemporary experiments, has in part been motivated by their simplicity in implementation. However, there are scenarios that benefit from expanding the system dimensionality, e.g., for enhancing information capacity, noise resilience, and robustness against external attacks in quantum cryptography $[1,2]$. Intrinsically high-dimensional degrees of freedom (DOFs) of light—such as orbital angular momentum (OAM), time, and frequency - enable a larger quantum alphabet in a single-photon state. The combination of two or more DOFs of light-including entanglement across them, namely, hyperentanglement $[3,4]$-allows further expansion of the Hilbert space while providing easy access to the individual subsystems for selective control and measurements, improving existing protocols or enabling new ones [5,6]. Hyperentanglement in particular enables protocols like complete Bell-state analysis [7-9] and logic gates simplification [10], and has

\footnotetext{
*Corresponding author: fraccalo@gmail.com

†Corresponding author: vincenzo.dambrosio@unina.it
}

Published by the American Physical Society under the terms of the Creative Commons Attribution 4.0 International license. Further distribution of this work must maintain attribution to the author(s) and the published article's title, journal citation, and DOI. been used in cluster state generation $[11,12]$ as well as in testing quantum foundations [13]. Moreover, hyperentangled systems have been successfully used for demonstrations of quantum dense coding [14] and teleportation of multiple DOFs of a single photon $[15,16]$.

Photonic hyperentangled states have been demonstrated in different encoding regimes, such as polarization, time and frequency bins, path, and OAM [4,9,17]. While entanglement of three DOFs has been demonstrated [4,18] the generation of hyperentanglement in spatially and spectrally structured light having nontrivial polarization and OAM patterns and temporal/spectral envelopes [19] (as discussed below in detail) remains elusive owing to the complexity of accessing such encoding regimes. Notably, structured light modeswhere one or more DOFs are modulated into custom light fields - are highly sought after in quantum photonics applications spanning communication, metrology, and imaging [19]. In this paper, we fill this gap combining a nonlinearity engineering technique $[20,21]$ with a spin-to-orbital-angularmomentum conversion scheme [22] to generate and characterize a biphoton state that exhibits complex entanglement between spectrally and spatially structured light. We produce hyperentanglement between time-frequency modes (TFMs) temporal/spectral envelopes of the electric field of the photons [6,21], and vector vortex beams (VVBs) — spatially structured beams characterized by a nonuniform polarization pattern on their transverse profile [23-25]. Due to their resilience to different noise sources, both TFMs [26,27] and VVBs $[5,28]$ are ideal encodings for free-space communication schemes. Meanwhile sources that generate polarization-TFM hyperentanglement could be immediately deployed in telecom networks, as both DOFs are suitable for long-distance 


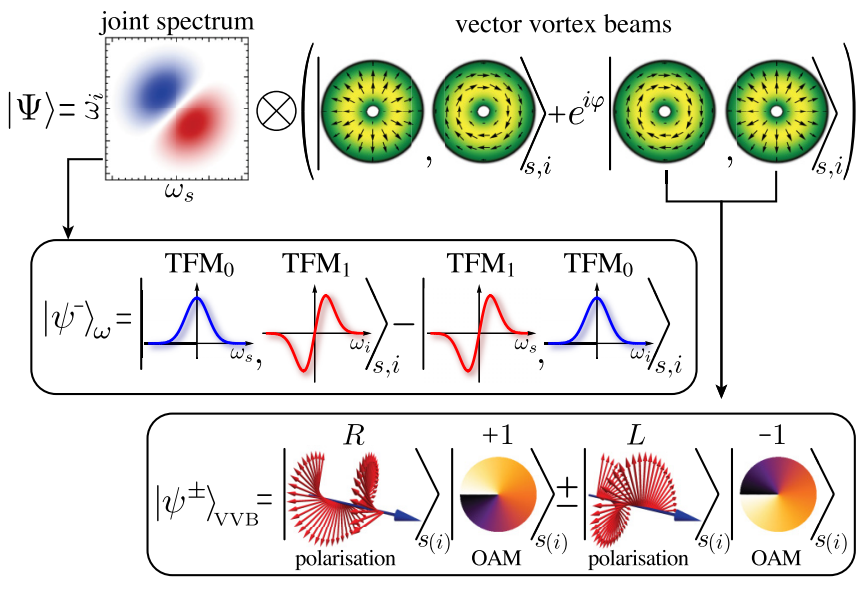

FIG. 1. Sketch of the biphoton hyperentangled state. The overall quantum state $|\Psi\rangle$ (first row) exhibits hyperentanglement between spectrally and spatially structured light. Signal and idler are encoded in the $\left|\psi^{-}\right\rangle_{\omega}$ state of the TFM basis, represented with the biphoton joint spectrum and the corresponding expansion in TFMs (first box), and in any Bell states of the VVB basis (we only display $|\psi\rangle$-type states for compactness). Each photon is also in a single-particle entangled state between polarization and OAM, giving rise to a VVB (second box). The plus (minus) sign corresponds to the radially (azimuthally) polarized beams, respectively.

transmission in fiber. Furthermore, a recent demonstration on quantum transmission of VVBs in specialized fibers [29] bolsters future prospects for fiber-based networks exploiting the full capabilities of our scheme. Finally, silicon based quantum photonics is compatible with periodically poled sources [30] and has been recently proven capable of generating spatially structured light [31], paving the way towards the integration of TFM-VVB hyperentanglement sources into photonics chips.

The generation of hyperentanglement between timefrequency modes and vector vortex beams implies the ability to independently create spectrally and spatially structured light. TFM encoding can be achieved via nonlinearity engineering, a technique that tailors the phase-matching function in parametric downconversion (PDC) processes by modifying the ferroelectric structure of periodically poled nonlinear crystals. Originally used for generating spectrally pure heralded single photons $[20,32,33]$, nonlinearity engineering has since been applied to generate TFM entanglement with high fidelity [21]. Vector vortex beams on the other hand can be efficiently created by converting polarization into polarization-OAM entanglement by means of birefringent liquid-crystal devices known as q-plates [22,24]. Our scheme combines these two techniques with an interferometric Sagnac scheme [34,35] for generating the highly entangled state with the nontrivial structure sketched in Fig. 1.

\section{HYPERENTANGLEMENT GENERATION}

We describe the experimental implementation of our state generation scheme in Fig. 2(a). A Ti-sapphire laser produces a train of near transform-limited, 1.3-ps pulses centered at $775 \mathrm{~nm}$ with $80-\mathrm{MHz}$ repetition rate. A plano-convex lens focuses the laser beam (30-mW average power) to a spot size


FIG. 2. Experimental setup. (a) State preparation: interferometric scheme to produce hyperentangled biphoton states in TFM and VVB encoding. (b) Characterization setup to measure the overall state via two-photon interference and tomographic reconstruction. A set of QWP, HWP, and QWP is used to prepare any maximally entangled state in the radial/azimuthal VVB basis. When the fast axis of the QWPs is aligned, a rotation of the HWP corresponds to changing the phase factor of the Bell-like state. The polarization projection stages in the dashed boxes, each consisting of the QWP, HWP, and polarizer, are used to perform projective measurements on the polarization of the photons for the 16-dimensional polarizationOAM biphoton state reconstruction.

of $\approx 220 \mu \mathrm{m}$ into a Sagnac interferometer PDC source for generating a polarization entangled state $[34,35]$ :

$$
\left|\psi^{-}\right\rangle_{\text {pol. }}=\frac{1}{\sqrt{2}}\left(|H\rangle_{s}|V\rangle_{i}-|V\rangle_{s}|H\rangle_{i}\right) .
$$

The PDC crystal in the Sagnac loop is a nonlinearity engineered potassium titanyl phosphate (KTP) poled crystal (details in Ref. [21]), that simultaneously enables the generation of TFM entanglement in a tailored PDC process. The phase-matching function of the engineered crystal is indeed shaped as a first-order Hermite-Gauss mode, leading to a highly correlated joint spectrum of the form $f_{s, i}\left(\omega_{s}, \omega_{i}\right) \propto$ $\exp \left[-\left(\omega_{s}^{2}+\omega_{i}^{2}\right)\right]$, which we show in Fig. 1, and consequently a TFM-entangled singlet state:

$$
\left|\psi^{-}\right\rangle_{\omega}=1 / \sqrt{2}\left(|\wedge\rangle_{s}|\sim\rangle_{i}-|\sim\rangle_{s}|\wedge\rangle_{i}\right),
$$

where $|\wedge\rangle$ and $|\sim\rangle$ are the orthonormal zeroth- and first-order Hermite-Gauss TFM modes spanning a twodimensional TFM subspace (see Sec. 1 of Supplemental Material for more details on the state generation [36]).

The PDC pair (signal "s" and idler "i") is separated at a polarizing beamsplitter (PBS), and the signal is filtered from the pump with a dichroic mirror (DM). The photons are then spectrally filtered with a long-pass filter (cutoff wavelength 
at $1400 \mathrm{~nm})$ and a "loose" bandpass filter (10-nm nominal bandwidth), about four times larger than the photons' bandwidth of $\approx 2.4 \mathrm{~nm}$, defined as the full width at half maximum of the marginal photon's spectral intensity. The photon pair is therefore coupled into single-mode fibers for spatial mode filtering. At this stage of the scheme, the quantum state carries hyperentanglement between the maximally antisymmetric TFM Bell state and a polarization Bell state, $|\Psi\rangle_{\omega \text {, pol. }}=$ $\left|\psi^{-}\right\rangle_{\omega} \otimes\left|\psi^{-}\right\rangle_{\text {pol. }}$, that is already potentially useful for fiberbased quantum photonic protocols, as both polarization and TFM are readily compatible with long-distance transmission over an optical network. We measure a source brightness of $\approx 4-\mathrm{KHz} / \mathrm{mW}$ detected photon pairs using superconducting nanowire single-photon detectors (SNSPDs) with $80 \%$ nominal detection efficiency, with symmetric heralding efficiency of $\approx 60 \%$. However, brightness and heralding can be easily varied by changing the focusing and collection parameters of the source.

The photons are then out-coupled and collimated in free space, and a set of quarter-wave plate (QWP), half-wave plate (HWP), and QWP is used to prepare any maximally entangled polarization state via local operations. Each photon is finally sent through a q-plate for converting polarization encoding into VVB encoding, producing the target TFM-VVB hyperentangled state. A q-plate with topological charge $q$ (with $q=0.5$ in our setup) implements the following transformation: $\alpha|R, 0\rangle+\beta|L, 0\rangle \rightarrow \alpha|L,-2 q\rangle+\beta|R, 2 q\rangle$ where the first and second label correspond to polarization and OAM value, respectively. If the input polarization is linear, a VVB in a linear superposition of the basis states $|\hat{r}\rangle$ (radially polarized) and $|\hat{\theta}\rangle$ (azimuthally polarized) is produced in the process. When each photon of the polarization-entangled biphoton state is sent through a q-plate, the overall system consists of two entangled vector vortex beams [37]. The q-plates used in our setup are electrically tunable, and have a transmittance of 70 to $75 \%$, that could be improved by coating the inner and outer surfaces of the devices, or by different engineering techniques (e.g., nontunable q-plates with nearunity transmission are already commercially available).

At the output of the q-plates, the biphoton state shows a nontrivial entanglement structure, where three different forms of entanglement coexist in the same quantum state: hyperentanglement between TFMs and VVBs, which is in turn composed of single-particle (intrasystem) entanglement [25,37-39] — polarization and OAM of each photon-and two distinct sets of intersystem entanglement-between the two VVBs and between the two TFMs, as sketched in Fig. 1 and shown in its full form below:

$$
|\Psi\rangle_{\omega, \mathrm{VVB}}=|\psi\rangle_{\omega} \otimes \underbrace{\frac{1}{\sqrt{2}}\left(|\hat{r}\rangle_{s}|\hat{\theta}\rangle_{i}-|\hat{\theta}\rangle_{s}|\hat{r}\rangle_{i}\right)}_{\left|\psi^{-}\right\rangle_{\mathrm{VVB}}},
$$

with $|\hat{r}\rangle_{\mathrm{vVB}}=\frac{1}{\sqrt{2}}\left(|R\rangle_{\mathrm{pol}}|+1\rangle_{\mathrm{OAM}}+|L\rangle_{\mathrm{pol}}|-1\rangle_{\mathrm{OAM}}\right)$ and $|\hat{\theta}\rangle_{\mathrm{VVB}}=$ $\frac{1}{\sqrt{2}}\left(|R\rangle_{\text {pol. }}|+1\rangle_{\text {OAM }}-|L\rangle_{\text {pol. }}|-1\rangle_{\text {OAM }}\right)$. We note that this scheme allows one to generate states within a two-dimensional VVB subspace of order 1, while additional HWPs after the q-plates enable the generation of any VVB state in the fourdimensional space [37].

\section{HYPERENTANGLEMENT CHARACTERIZATION}

The analysis stage consists of two main steps, as shown in Fig. 2(b). First, we send the two photons on a BS to check for quantum interference depending on the symmetry of the full state [21]. After the BS, a set of two additional q-plates (with $q=0.5$ ) and polarization optics (QWP, HWP, and PBS for each photon) are used to perform tomographic projections in the VVB space, and the photons are finally detected with SNSPDs. An additional tomographic projection set can be added before the measurement q-plates to perform a four-qubit tomography in the polarization and OAM subspaces, simultaneously certifying the intrasystem entangled structure of each VVB and the intersystem entanglement between the two photons [37], and verifying the GreenbergerHorne-Zeilinger (GHZ)-type structure of the state [40].

The synergy of the quantum interference and tomographic parts of the setup allows us to demonstrate the hyperentangled nature of the biphoton state. The tomographic stages are decoupled from the spectral degree of freedom and only act on the polarization and OAM components of the state, providing full reconstruction of the VVB-encoded quantum state: this means that our setup is capable of preparing and measuring unambiguously the spatial component of the state. On the other hand, while the visibility depends on the overall state's symmetry, the shape of the interference pattern only depends on the biphoton joint spectrum (see Sec. 2 of Supplemental Material for proof [36]). Consequently, the knowledge of the VVB component of the state combined with the measured interference pattern allows us to decouple spatial and spectral contributions of the overall measurement, and to use the interference results to certify the TFM entanglement, as proven in Ref. [21].

\section{RESULTS}

To benchmark our scheme, we produce states of the form

$$
|\Psi\rangle_{\omega, \mathrm{VVB}}=\left|\psi^{-}\right\rangle_{\omega} \otimes\left|\psi^{\varphi}\right\rangle_{\mathrm{VVB}}
$$

where $\left|\psi^{\varphi}\right\rangle_{\mathrm{VVB}}=1 / \sqrt{2}\left(|\hat{r}, \hat{\theta}\rangle+e^{i \varphi}|\hat{\theta}, \hat{r}\rangle\right)$ is a $\psi$-type maximally entangled state in the VVB basis. By changing the state preparation HWP angle we can change the phase $\varphi$ of the VVB part of the state and hence the symmetry of the overall wave function. The phase factors $e^{i 0}$ and $e^{i \pi}$ correspond to the $\left|\psi^{-}\right\rangle_{\omega} \otimes\left|\psi^{+}\right\rangle_{\mathrm{VVB}}$ and $\left|\psi^{-}\right\rangle_{\omega} \otimes\left|\psi^{-}\right\rangle_{\mathrm{VVB}}$ states, i.e., to a maximally antisymmetric and symmetric state, respectively. This translates into different interference behavior at the BS, where moving from an antisymmetric to a symmetric state corresponds to moving from photon antibunching to photon bunching, as we show in Fig. 3. When the spatial contribution is symmetric $\left(\left|\psi^{+}\right\rangle_{\mathrm{VVB}}\right)$ the interference exhibits perfect antibunching, while when it is antisymmetric $\left(\left|\psi^{-}\right\rangle_{\mathrm{VVB}}\right)$ it exhibits bunching: this certifies the maximal antisymmetry of the spectral part of the state, and consequently verifies the TFM singlet state $\left|\psi^{-}\right\rangle_{\omega}$ (see Ref. [21] for details).

We monitor coincident counts between detectors $\left\{\mathrm{A}_{1}, \mathrm{~B}_{1}\right\}$, $\left\{A_{1}, B_{2}\right\},\left\{A_{2}, B_{1}\right\}$, and $\left\{A_{2}, B_{2}\right\}$, corresponding to the photons exiting from both outputs of the $\mathrm{BS}$, and between the detectors $\left\{A_{1}, A_{2}\right\}$ and $\left\{B_{1}, B_{2}\right\}$, corresponding to the photons exiting the same outputs of the $\mathrm{BS}$, to reconstruct the 
(a)
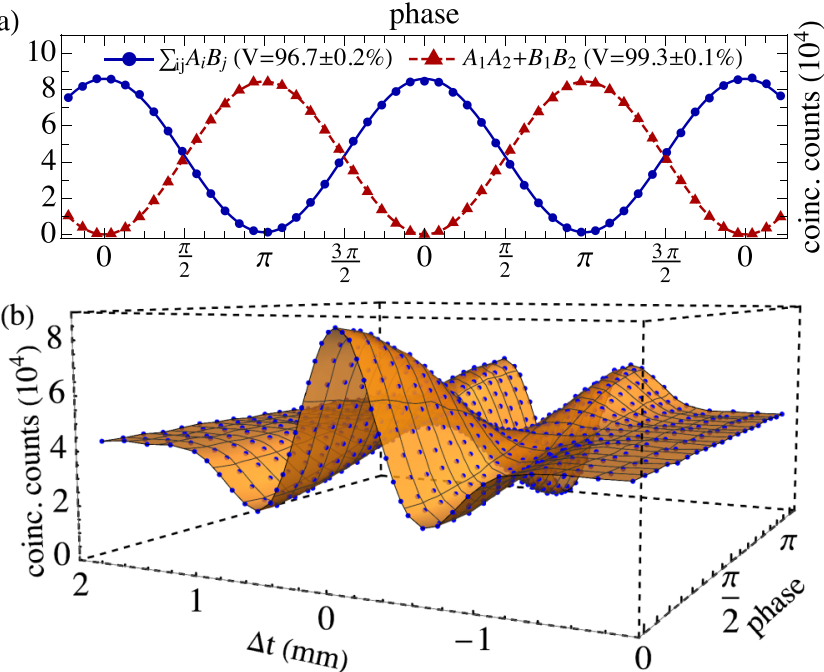

(c) 1

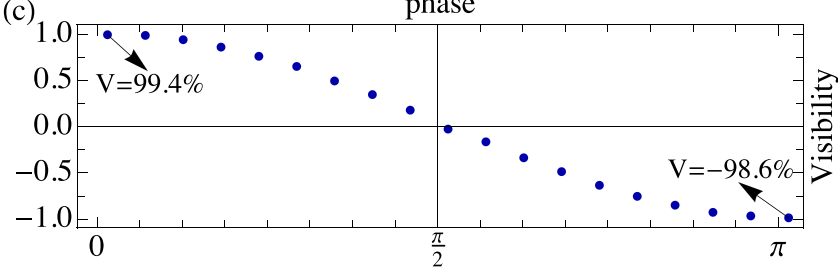

FIG. 3. Two-photon interference results. (a) The interference fringes depend on the phase in the VVB-encoded part of the hyperentangled state: A maximum in the coincident counts at the two outputs of the BS (labeled as $\sum_{i j} A_{i} B_{j}$, where $A_{i}, B_{j}$ are the detectors in Fig. 2) corresponds to a minimum in the coincident counts at each BS output (labeled as $A_{1} A_{2}+B_{1} B_{2}$ ), and vice versa. Interference patterns measured by collecting coincident counts at the two outputs of the BS (b) and corresponding visibilities (c) changing phase and relative arrival time of the photons at the BS. By controlling the phase of the $|\psi\rangle$ type we can move from almost perfect antibunching to bunching, i.e., from an overall antisymmetric state to a symmetric one. The acquisition time is $30 \mathrm{~s}$ per data point.

interference fringes as a function of the state's phase $\varphi$. We show the results in Fig. 3(a): the fringes corresponding to antibunching (blue dots) and to bunching (red triangles) are in antiphase, and have high visibilities (96.7 \pm 0.2 and $99.3 \pm 0.1 \%$, respectively) certifying a high quality of the generated state. By varying both the state's phase and the relative arrival time of signal and idler at the BS, we can reconstruct the full biphoton interference pattern for states with different amounts of antisymmetry. The three-dimensional plot in Fig. 3(b) shows how the interference pattern changes from perfect antibunching (corresponding to $\varphi=0$ ) to perfect bunching $(\varphi=\pi)$, in excellent agreement with the theoretical model we discuss in Sec. 2 of Supplemental Material [36]. Finally, Fig. 3(c) shows the interference visibilities of each scan, where plus and minus $100 \%$ correspond to perfect antibunching and bunching, respectively.

The two-photon interference allows us to measure the overall antisymmetry of the biphoton state, but it does not provide any information on its spatial structure. The VVB state can instead be measured via quantum state tomography after the interference at the BS. We prepare the symmetric state
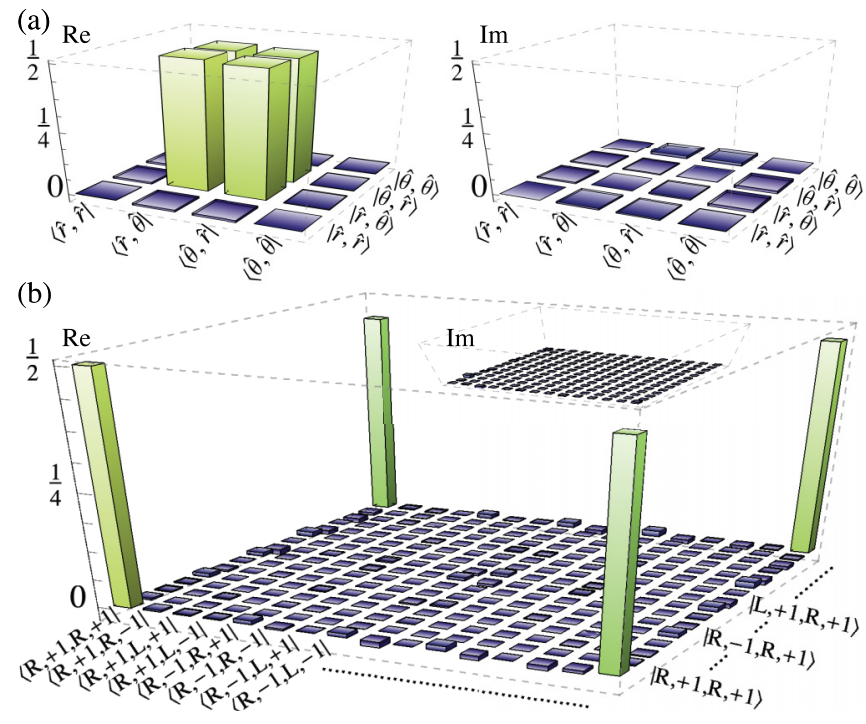

FIG. 4. Tomography results. (a) Tomographic reconstruction of the biphoton $\left|\psi^{+}\right\rangle$state in the VVB subspace $\{|\hat{r}\rangle,|\hat{\theta}\rangle\}$. We record $\approx 1.6 \times 10^{6}$ coincident counts in nine different settings (corresponding to the two-photon Pauli projections), with $60 \mathrm{~s}$ of acquisition time per setting. (b) Tomographic reconstruction of the biphoton GHZ state encoded in polarization and OAM. We record $\approx 11 \times 10^{6}$ coincident counts in 324 different settings (corresponding to a combination of 36 projective measurements on the OAM basis and nine Pauli projections on the polarization basis), with $220 \mathrm{~s}$ of acquisition time per setting.

$\left|\psi^{-}\right\rangle_{\omega} \otimes\left|\psi^{+}\right\rangle_{\mathrm{VVB}}$, which antibunches at the BS. We then convert the VVB information into polarization information, and we perform a overcomplete quantum state tomography of the state. We measure a purity and fidelity of $(99.26 \pm 0.07)$ and $(99.57 \pm 0.03) \%$, respectively, in the VVB subspace $\{|\hat{r}\rangle,|\hat{\theta}\rangle\}$ [see Fig. 4(a)]. Introducing an additional tomographic projection before each measurement q-plate allows us to investigate the polarization-OAM intrasystem entanglement and, at the same time, the two-photon intersystem entanglement [37]. With this scheme, we measure a two-photon, four-qubit purity of $(92.4 \pm 0.1) \%$ and a fidelity of $(95.0 \pm 0.1) \%$ with the GHZ state $1 / \sqrt{2}(|R,+1, R,+1\rangle+|L,-1, L,-1\rangle)$ : we show the corresponding density matrix in Fig. 4(b). Errors on the extracted purities and fidelities calculated from the reconstructed density matrices represent $3 \sigma$ statistical confidence regions obtained via Monte Carlo resampling (1000 runs of the algorithm) assuming a Poissonian statistics on the coincident counts distribution.

The high interference visibility measured in both the bunching and antibunching configuration, combined with the high state quality obtained via tomographic reconstruction of the VVB-encoded state, testifies to an unprecedented capability of generating and manipulating structured light encoded in three different degrees of freedom with very high efficiency and precision.

\section{DISCUSSION AND CONCLUSIONS}

Many photonic quantum protocols rely on entanglement to carry out their tasks efficiently, therefore the capability 
of generating and manipulating complex entangled states of light with high precision is a fundamental requirement and a key challenge of quantum technologies. Here, we tackled this problem by introducing and experimentally demonstrating a scheme for efficient generation of a complex entanglement structure between three DOFs of light: polarization, orbital angular momentum, and time-frequency modes. In particular, we combine TFM encoding and VVB encoding introducing a simple yet high-quality source of TFM-VVB hyperentanglement. We expect our scheme will find applications in quantum communication schemes (where increased information capacity and noise resilience are obvious advantages) but also in different areas of quantum technologies, such as metrology or imaging, where both TFM and VVB encoding have already been independently used as resources $[41,42]$.

There are two main routes to go beyond the results of this paper in the future. On the one hand, it would be ideal to explore the intrinsic high dimensionality of these DOFs, generating higher-order OAM and TFM states to increase the information capacity of the biphoton state and investigate even more complex entanglement structures. This could be achieved by expanding either the TFM subspace (e.g., by engineering higher-order Hermite-Gauss modes as nonlinearity patterns, or by introducing spectral tailoring of the pump's spectral profile) or the VVB subspace (e.g., using q-plates of different singularity order in interferometric schemes), or a combination of the two. On the other hand, implementing quantum pulse gates or other schemes [6,43-45] for performing TFM manipulation and measurements would allow one to fully exploit the potential of our technique.

\section{ACKNOWLEDGMENTS}

This work was supported by the Engineering and Physical Sciences Research Council (EPSRC Grants No. EP/N002962/1, No. EP/T001011/1, and No. EP/L015110/1); the Italian Ministry of Education, University, and Research through the PRIN Project INPhoPOL; and the European Union Horizon 2020 program, within European Research Council Grant No. 694683, PHOSPhOR.
[1] M. Erhard, R. Fickler, M. Krenn, and A. Zeilinger, Twisted photons: New quantum perspectives in high dimensions, Light: Science \& Applications 7, 17146 (2018).

[2] D. Cozzolino, B. Da Lio, D. Bacco, and L. K. Oxenløwe, Highdimensional quantum communication: Benefits, progress, and future challenges, Adv. Quantum Technol. 2, 1900038 (2019).

[3] P. G. Kwiat, Hyper-entangled states, J. Mod. Opt. 44, 2173 (1997).

[4] J. T. Barreiro, N. K. Langford, N. A. Peters, and P. G. Kwiat, Generation of Hyperentangled Photon Pairs, Phys. Rev. Lett. 95, 260501 (2005).

[5] V. D'Ambrosio, E. Nagali, S. P. Walborn, L. Aolita, S. Slussarenko, L. Marrucci, and F. Sciarrino, Complete experimental toolbox for alignment-free quantum communication, Nat. Commun. 3, 961 (2012).

[6] B. Brecht, D. V. Reddy, C. Silberhorn, and M. G. Raymer, Photon temporal modes: A complete framework for quantum information science, Phys. Rev. X 5, 041017 (2015).

[7] S. P. Walborn, S. Pádua, and C. H. Monken, Hyperentanglement-assisted Bell-state analysis, Phys. Rev. A 68, 042313 (2003).

[8] C. Schuck, G. Huber, C. Kurtsiefer, and H. Weinfurter, Complete Deterministic Linear Optics Bell State Analysis, Phys. Rev. Lett. 96, 190501 (2006).

[9] M. Barbieri, G. Vallone, P. Mataloni, and F. De Martini, Complete and deterministic discrimination of polarization Bell states assisted by momentum entanglement, Phys. Rev. A 75, 042317 (2007).

[10] B. P. Lanyon, M. Barbieri, M. P. Almeida, T. Jennewein, T. C. Ralph, K. J. Resch, G. J. Pryde, J. L. O’Brien, A. Gilchrist, and A. G. White, Simplifying quantum logic using higherdimensional hilbert spaces, Nat. Phys. 5, 134 (2009).

[11] G. Vallone, G. Donati, R. Ceccarelli, and P. Mataloni, Six-qubit two-photon hyperentangled cluster states: Characterization and application to quantum computation, Phys. Rev. A 81, 052301 (2010).
[12] M. A. Ciampini, A. Orieux, S. Paesani, F. Sciarrino, G. Corrielli, A. Crespi, R. Ramponi, R. Osellame, and P. Mataloni, Path-polarization hyperentangled and cluster states of photons on a chip, Light: Science \& Applications 5, e16064 (2016).

[13] M. Barbieri, F. De Martini, P. Mataloni, G. Vallone, and A. Cabello, Enhancing the Violation of the Einstein-PodolskyRosen Local Realism by Quantum Hyperentanglement, Phys. Rev. Lett. 97, 140407 (2006).

[14] J. T. Barreiro, T.-C. Wei, and P. G. Kwiat, Beating the channel capacity limit for linear photonic superdense coding, Nat. Phys. 4, 282 (2008).

[15] Y.-B. Sheng, F.-G. Deng, and G. L. Long, Complete hyperentangled-Bell-state analysis for quantum communication, Phys. Rev. A 82, 032318 (2010).

[16] X.-L. Wang, X.-D. Cai, Z.-E. Su, M.-C. Chen, D. Wu, L. Li, N.-L. Liu, C.-Y. Lu, and J.-W. Pan, Quantum teleportation of multiple degrees of freedom of a single photon, Nature (London) 518, 516 (2015).

[17] Z. Xie, T. Zhong, S. Shrestha, X. Xu, J. Liang, Y.-X. Gong, J. C. Bienfang, A. Restelli, J. H. Shapiro, F. N. C. Wong, and C. Wei Wong, Harnessing high-dimensional hyperentanglement through a biphoton frequency comb, Nat. Photonics 9, 536 (2015).

[18] X.-L. Wang, Y.-H. Luo, H.-L. Huang, M.-C. Chen, Z.-E. Su, C. Liu, C. Chen, W. Li, Y.-Q. Fang, X. Jiang, J. Zhang, L. Li, N.-L. Liu, C.-Y. Lu, and J.-W. Pan, 18-Qubit Entanglement with Six Photons' Three Degrees of Freedom, Phys. Rev. Lett. 120, 260502 (2018).

[19] H. Rubinsztein-Dunlop, A. Forbes, M. V. Berry, M. R. Dennis, D. L. Andrews, M. Mansuripur, C. Denz, C. Alpmann, P. Banzer, T. Bauer, E. Karimi, L. Marrucci, M. Padgett, M. Ritsch-Marte, N. M. Litchinitser, N. P. Bigelow, C. RosalesGuzmán, A. Belmonte, J. P. Torres, T. W. Neely, M. Baker, R. Gordon, A. B. Stilgoe, J. Romero, A. G. White, R. Fickler, A. E. Willner, G. Xie, B. McMorran, and A. M. Weiner, Roadmap on structured light, J. Opt. 19, 013001 (2016). 
[20] F. Graffitti, D. Kundys, D. T. Reid, A. M. Brańczyk, and A. Fedrizzi, Pure down-conversion photons through subcoherence-length domain engineering, Quantum Sci. Technol. 2, 035001 (2017).

[21] F. Graffitti, P. Barrow, A. Pickston, A. M. Brańczyk, and A. Fedrizzi, Direct Generation of Tailored Pulse-Mode Entanglement, Phys. Rev. Lett. 124, 053603 (2020).

[22] L. Marrucci, C. Manzo, and D. Paparo, Optical Spinto-Orbital Angular Momentum Conversion in Inhomogeneous Anisotropic Media, Phys. Rev. Lett. 96, 163905 (2006).

[23] M. R. Dennis, K. O'Holleran, and M. J. Padgett, Chapter 5 Singular Optics: Optical Vortices and Polarization Singularities (Elsevier, Amsterdam, 2009), pp. 293-363.

[24] F. Cardano, E. Karimi, S. Slussarenko, L. Marrucci, C. de Lisio, and E. Santamato, Polarization pattern of vector vortex beams generated by q-plates with different topological charges, Appl. Opt. 51, C1 (2012).

[25] A. Selyem, C. Rosales-Guzmán, S. Croke, A. Forbes, and S. Franke-Arnold, Basis-independent tomography and nonseparability witnesses of pure complex vectorial light fields by Stokes projections, Phys. Rev. A 100, 063842 (2019).

[26] A. Eckstein, B. Brecht, and C. Silberhorn, A quantum pulse gate based on spectrally engineered sum frequency generation, Opt. Express 19, 13770 (2011).

[27] Q. Ding, R. Chatterjee, Y. Huang, and T. Yu, High-dimensional temporal mode propagation in a turbulent environment, arXiv:1907.02321 (2019).

[28] O. J. Farías, V. D’Ambrosio, C. Taballione, F. Bisesto, S. Slussarenko, L. Aolita, L. Marrucci, S. P. Walborn, and F. Sciarrino, Resilience of hybrid optical angular momentum qubits to turbulence, Sci. Rep. 5, 8424 (2015).

[29] D. Cozzolino, E. Polino, M. Valeri, G. Carvacho, D. Bacco, N. Spagnolo, L. K. K. Oxenløwe, and F. Sciarrino, Air-core fiber distribution of hybrid vector vortex-polarization entangled states, Adv. Photonics 1, 046005 (2019).

[30] S. Atzeni, A. S. Rab, G. Corrielli, E. Polino, M. Valeri, P. Mataloni, N. Spagnolo, A. Crespi, F. Sciarrino, and R. Osellame, Integrated sources of entangled photons at the telecom wavelength in femtosecond-laser-written circuits, Optica 5, 311 (2018).

[31] Y. Chen, K.-Y. Xia, W.-G. Shen, J. Gao, Z.-Q. Yan, Z.-Q. Jiao, J.-P. Dou, H. Tang, Y.-Q. Lu, and X.-M. Jin, Vector Vortex Beam Emitter Embedded in a Photonic Chip, Phys. Rev. Lett. 124, 153601 (2020)
[32] A. M. Brańczyk, A. Fedrizzi, T. M. Stace, T. C. Ralph, and A. G. White, Engineered optical nonlinearity for quantum light sources, Opt. Express 19, 55 (2011).

[33] F. Graffitti, P. Barrow, M. Proietti, D. Kundys, and A. Fedrizzi, Independent high-purity photons created in domain-engineered crystals, Optica 5, 514 (2018).

[34] T. Kim, M. Fiorentino, and F. N. C. Wong, Phase-stable source of polarization-entangled photons using a polarization Sagnac interferometer, Phys. Rev. A 73, 012316 (2006).

[35] A. Fedrizzi, T. Herbst, A. Poppe, T. Jennewein, and A. Zeilinger, A wavelength-tunable fiber-coupled source of narrowband entangled photons, Opt. Express 15, 15377 (2007).

[36] See Supplemental Material at http://link.aps.org/supplemental/ 10.1103/PhysRevResearch.2.043350 for details on the state generation and characterization schemes.

[37] V. D’Ambrosio, G. Carvacho, F. Graffitti, C. Vitelli, B. Piccirillo, L. Marrucci, and F. Sciarrino, Entangled vector vortex beams, Phys. Rev. A 94, 030304(R) (2016).

[38] A. Aiello, F. Töppel, C. Marquardt, E. Giacobino, and G. Leuchs, Quantum-like nonseparable structures in optical beams, New J. Phys. 17, 043024 (2015).

[39] M. McLaren, T. Konrad, and A. Forbes, Measuring the nonseparability of vector vortex beams, Phys. Rev. A 92, 023833 (2015).

[40] G. Carvacho, F. Graffitti, V. D’Ambrosio, B. C. Hiesmayr, and F. Sciarrino, Experimental investigation on the geometry of GHZ states, Sci. Rep. 7, 13265 (2017).

[41] V. D’Ambrosio, N. Spagnolo, L. Del Re, S. Slussarenko, Y. Li, L. C. Kwek, L. Marrucci, S. P. Walborn, L. Aolita, and F. Sciarrino, Photonic polarization gears for ultra-sensitive angular measurements, Nat. Commun. 4, 2432 (2013).

[42] J. M. Donohue, V. Ansari, J. Řeháček, Z. Hradil, B. Stoklasa, M. Paúr, L. L. Sánchez-Soto, and C. Silberhorn, QuantumLimited Time-Frequency Estimation through Mode-Selective Photon Measurement, Phys. Rev. Lett. 121, 090501 (2018).

[43] Y.-P. Huang and P. Kumar, Mode-resolved photon counting via cascaded quantum frequency conversion, Opt. Lett. 38, 468 (2013).

[44] V. Ansari, J. M. Donohue, M. Allgaier, L. Sansoni, B. Brecht, J. Roslund, N. Treps, G. Harder, and C. Silberhorn, Tomography and Purification of the Temporal-Mode Structure of Quantum Light, Phys. Rev. Lett. 120, 213601 (2018).

[45] D. V. Reddy and M. G. Raymer, High-selectivity quantum pulse gating of photonic temporal modes using all-optical Ramsey interferometry, Optica 5, 423 (2018). 